\title{
Efficient Multiple Access Protocols for Coded Multi-Source Multi-Relay Networks
}

\author{
Chao Wang, Ming Xiao, and Mikael Skoglund \\ School of Electrical Engineering, Royal Institute of Technology (KTH), Stockholm, Sweden \\ Emails: \{Chaowang, Ming.Xiao, Skoglund\}@ee.kth.se
}

\begin{abstract}
We study the impact of multiple access protocols on the diversity-multiplexing tradeoff (DMT) performance in wireless multi-user relay networks. In the networks $K$ halfduplex decode-and-forward (DF) relays employ a class of finite field network codes to assist in the communication between $M$ independent sources and a common destination. The sources and relays are divided into individual clusters. The nodes within one cluster transmit non-orthogonally while the transmissions of different clusters span orthogonal channels. We provide the method to calculate the achievable DMT for each clustering strategy. The network DMT performance can thus be optimized by properly clustering the sources and the relays.
\end{abstract}

\section{INTRODUCTION}

Relaying has been considered as an effective approach to provide multi-path diversity in wireless networks (e.g. [1], [2]). By introducing intermediate relays to assist in the communication between a source-destination pair, the transmit messages can be delivered via multiple independent paths so that the negative impact of fading can be significantly reduced.

In multi-user decode-and-forward (DF) relay networks, employing network coding [3], [4] on top of channel coding has attracted much interest in the last few years due to its high spectral and power efficiency compared to conventional approaches without network coding. For instance, consider a four-node network in which two sources intend to send independent messages $I_{S_{1}}$ and $I_{S_{2}}$ respectively to a common destination through the help of a relay. Instead of separately forwarding $I_{S_{1}}$ and $I_{S_{2}}$, the relay can apply the conventional binary network code (BNC) to efficiently transmit both messages simultaneously, i.e. $I_{S_{1}} \bigoplus I_{S_{2}}$ (addition in Galois field $\mathrm{GF}(2)$ ), to the destination (e.g. [5] [6]). Thus potentially the source message set $\mathcal{I}_{S}=\left\{I_{S_{1}}, I_{S_{2}}\right\}$ is delivered to the destination through three independent paths: two direct sourcedestination links and one multi-hop relay link. The destination cannot recover $\mathcal{I}_{S}$ (we say the transmission fails) only if the link conditions of at least two links are not good enough. This implies an achievable diversity gain 2 for each source, when the signal-to-noise ratio (SNR) approaches infinity and the transmission data rate does not change with changing SNR.

Nevertheless, adopting the BNC in multi-source multi-relay networks may not be diversity optimal. For example, when an extra relay is introduced into the above two-source network, requiring both relays to forward $I_{S_{1}} \bigoplus I_{S_{2}}$ provides only diversity gain 2 for fixed transmission rate although potentially the sources can be protected by two relays [7], [8]. This is because that two links among the two direct links and the two relay links are not good enough may lead to a failed transmission: the destination cannot recover $\mathcal{I}_{S}$ from only the relays' messages $I_{S_{1}} \bigoplus I_{S_{2}}$. To handle this issue, a new design of network codes [7], [8] requires the two relays to add $I_{S_{1}}$ and $I_{S_{2}}$ in $\mathrm{GF}(4)$ and generate different messages $I_{S_{1}}+I_{S_{2}}$ and $I_{S_{1}}+2 I_{S_{2}}$ respectively. $\mathcal{I}_{S}$ can thus be recovered as long as any two of the four links are sufficiently good. When the transmission rate is fixed, the expected diversity 3 is achieved.

Such a finite field network coding design can be readily extended to a general network with $M$ sources and $K$ relays. If the $j$ th relay successfully decodes all source messages, it adds them in a certain finite field to generate a new transmit message $\sum_{i=1}^{M} \gamma_{j, i} I_{S_{i}}$, where the coding coefficients $\gamma_{j, i}$ are designed in such a way that the global encoding kernels (GEK) [9] are linearly independent. The transmission fails only when the link conditions of at least $K+1$ links among the $M$ direct source-destination links and $K$ multi-hop relay links are not good enough. The diversity gain for fixed transmission rate $K+1$ is obtained. Since this class of network codes have the maximum distance separable (MDS) code structure [10], we term them MDS finite field network codes (MDS-FFNCs). It is shown in [7] and [8] that such network codes always exist if the coding field size is sufficiently large.

In this paper, we also consider the $M$-source $K$-relay single-destination network. The class of MDS-FFNCs are employed in the relays. This paper focuses on the impact of physical layer multiple access (MA) protocols on diversity performance. To this end, we define the diversity gain $d$ and multiplexing gain $r$ as [11]

$$
d=-\lim _{\rho \rightarrow \infty} \frac{\log P_{e}(\rho)}{\log \rho} \quad \text { and } \quad r=\lim _{\rho \rightarrow \infty} \frac{\bar{R}(\rho)}{\log \rho},
$$

where $\rho$ denotes SNR, $P_{e}$ denotes the error probability and $\bar{R}$ denotes the transmission rate in bits per channel use (BPCU). We use the diversity-multiplexing tradeoff (DMT) to evaluate transmission protocols from both communication reliability and spectral efficiency aspects.

Further, fully orthogonal transmission among sources and relays is commonly assumed in previous work on applying network coding in relay networks (including [7] and [8]). We propose a more general transmission protocol that divides the sources and the relays into non-overlapping clusters. The nodes within one cluster transmit their messages nonorthogonally (i.e. non-orthogonal MA within one cluster) while the transmissions of different clusters span orthogonal 


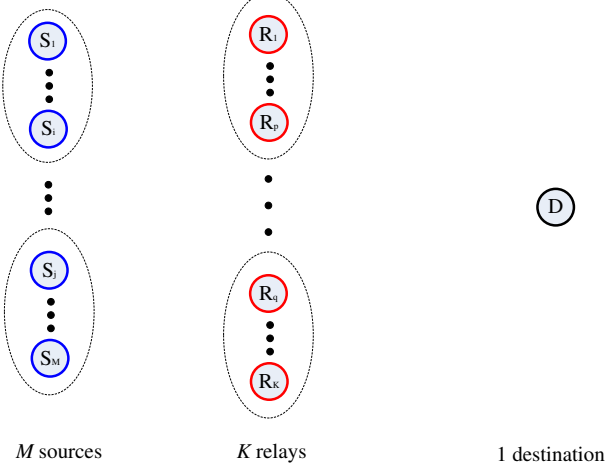

Fig. 1. The multi-source multi-relay network model. The $M$ sources are divided into $m$ clusters and the $K$ relays are divided into $k$ clusters.

channels (i.e. orthogonal MA across different clusters). For different values of multiplexing gain, we show that the diversity performances can be optimized respectively by different proper clustering/MA strategies.

Notations: We use $\log (\cdot)$ to denote the base-2 logarithm. The complement set and the cardinality of set $\mathcal{A}$ are denoted as $\mathcal{A}^{c}$ and $|\mathcal{A}|$ respectively. $(x)^{+}$means $\max \{0, x\}$.

\section{System Model And Network Codes Design}

We consider a single-antenna wireless network where the communication between $M(M \geq 2)$ sources $S_{1}, \cdots, S_{M}$ and a common destination $D$ is assisted by $K(K \geq 1)$ halfduplex DF relays $R_{1}, \cdots, R_{K}$, as displayed in Fig. 1. The $M$ sources intend to send independent messages $I_{S_{1}}, \cdots, I_{S_{M}}$ respectively to $D$. The source message set is denoted as $\mathcal{I}_{S}=$ $\left\{I_{S_{1}}, I_{S_{2}}, \cdots, I_{S_{M}}\right\}$.

A slow, flat Rayleigh fading environment is assumed, where channel fading coefficients are independent, identically distributed (i.i.d.) random variables for different channels but constant for the whole transmission period. A narrow-band transmission is considered such that all nodes transmit in the same frequency band. Each message's transmission spans a unit channel (one time slot). No cooperation among sources exists and the channel state information (CSI) is known at only the receiver of each link. Moreover, we assume a symmetric network such that each terminal transmits with equal power and equal transmission rate.

Due to the practical half-duplex limitation, the transmission consists of two orthogonal phases. During the first broadcasting phase, the sources broadcast their messages (using appropriate channel codes ${ }^{1}$ ) to the relays and $D$. The relays try to decode the sources. If relay $R_{j}(1 \leq j \leq K)$ successfully decodes the source message set $\mathcal{I}_{S}$ (we say $R_{j}$ is in the decoding relay set $\mathcal{R}$ ), it adds all the source messages in a certain finite field and generate a new message (i.e. network codeword) $I_{R_{j}}=\sum_{i=1}^{M} \gamma_{j, i} I_{S_{i}}$, where $\gamma_{j, i}$ is the coding coefficient for $I_{S_{i}}$. Then during the second forwarding phase,

\footnotetext{
${ }^{1}$ In this paper, we assume that the channel codes employed on the physical layer are sufficiently strong such that the decoding error probability can be made sufficiently small, when the channel is not in outage. If the channel is in outage, the receiver cannot decode without errors. Consequently, the overall error performance is actually dominated by the outage performance [11].
}

the relays within $\mathcal{R}$ transmit their messages (using appropriate channel codes) to $D$ while the sources and the relays which are not in $\mathcal{R}$ are silent. Equivalently, the linear relation between the potential transmit message of each node and $\mathcal{I}_{S}$ is

$$
\left[\begin{array}{c}
I_{S_{1}} \\
I_{S_{2}} \\
\vdots \\
I_{S_{M}} \\
I_{R_{1}} \\
I_{R_{2}} \\
\vdots \\
I_{R_{K}}
\end{array}\right]=\underbrace{\left[\begin{array}{cccc}
1 & 0 & \cdots & 0 \\
0 & 1 & \cdots & 0 \\
\vdots & \vdots & \ddots & \vdots \\
0 & 0 & \cdots & 1 \\
\gamma_{1,1} & \gamma_{1,2} & \cdots & \gamma_{1, M} \\
\gamma_{2,1} & \gamma_{2,2} & \cdots & \gamma_{2, M} \\
\vdots & \vdots & \ddots & \vdots \\
\gamma_{K, 1} & \gamma_{K, 2} & \cdots & \gamma_{K, M}
\end{array}\right]}_{\mathbf{G}}\left[\begin{array}{c}
I_{S_{1}} \\
I_{S_{2}} \\
\vdots \\
I_{S_{M}}
\end{array}\right] .
$$

The network codes are designed in such a way that a submatrix constituted by any $M$ rows of the transfer matrix $\mathbf{G}$ is non-singular so that $\mathcal{I}_{S}$ can be recovered given any $M$ elements in set $\left\{I_{S_{1}}, \cdots, I_{S_{M}}, I_{R_{1}}, \cdots, I_{R_{K}}\right\}$ [7], [8]. This class of network codes are termed MDS-FFNCs. $D$ tries to decode all the messages it received from the $M$ sources and $|\mathcal{R}|$ relays. As long as any $M$ transmitting nodes' messages are correctly decoded, $D$ is able to recover the source message set $\mathcal{I}_{S}$. Otherwise, the transmission fails.

In most previous work on applying network coding in multihop relay networks, all the sources and relays are assumed to transmit orthogonally. In this paper, however, we relax this assumption and propose a more general transmission protocol. Specifically, we divide the sources and relays into individual clusters. The nodes within the same cluster transmit their messages non-orthogonally within the same channel, while the transmissions of different clusters span orthogonal channels. We term this novel protocol the cluster MDS-FFNC protocol and provide the detailed description and analysis in the following sections.

\section{PROTOCOL DESCRIPTION}

The $M$ sources are divided into $m$ non-overlapping clusters $(1 \leq m \leq M)$. Each source cluster $\mathcal{S}_{i}$ contains $M_{i}$ sources $\left(\sum_{i=1}^{m} M_{i}=M\right)$. The $K$ relays are divided into $k$ nonoverlapping clusters and each cluster $\mathcal{R}_{i}$ contains $K_{i}$ relays $\left(1 \leq k \leq K\right.$ and $\left.\sum_{i=1}^{k} K_{i}=K\right)$. Without loss of generality, we assume $M_{1} \geq M_{2} \geq \cdots \geq M_{m}$ and $K_{1} \geq K_{2} \geq \cdots \geq$ $K_{k}$.

A total of $m+k$ time slots are used to finish the transmission of $\mathcal{I}_{S}$. We assume each node to transmit with rate $R=(m+$ k) $\bar{R}$ bits per message so that the average transmission rate for each source is $\bar{R}$ BPCU. During the $p$ th time slot $(1 \leq p \leq$ $m$ ), the sources within cluster $\mathcal{S}_{p}$ broadcast their messages simultaneously. The received signals at relay $R_{j}(1 \leq j \leq K)$ and the destination $D$ are expressed as

$$
\begin{gathered}
y_{R_{j}}[p]=\sum_{S_{l} \in \mathcal{S}_{p}} \sqrt{\rho} h_{S_{l}, R_{j}} x_{S_{l}}+n_{R_{j}}[p], \\
y_{D}[p]=\sum_{S_{l} \in \mathcal{S}_{p}} \sqrt{\rho} h_{S_{l}, D} x_{S_{l}}+n_{D}[p],
\end{gathered}
$$


where $h_{a, b}$ is the channel fading coefficient between nodes $a$ and $b, x_{a}$ is the transmit signal from node $a, y_{b}[i]$ and $n_{b}[i]$ are the received signal and the additive white Gaussian noise (AWGN) at node $b$ during time slot $i$ respectively.

During each time slot, relay $R_{j}$ applies successive interference cancellation (SIC) to decode the mutually interfered sources. In other words, $R_{j}$ first decodes only the strongest signal while treating the other signals as Gaussian noise. Then the successfully decoded signal is subtracted before $R_{j}$ performs the similar process to decode the other sources' signals. If $R_{j}$ successfully decodes the whole source message set $\mathcal{I}_{S}$ (i.e. $R_{j} \in \mathcal{R}$ ), it generates its transmit message $I_{R_{j}}$ according to (2). The next $k$ time slots after the broadcasting phase are allocated to the $k$ relay clusters respectively. During the $(m+q)$ th time slot $(1 \leq q \leq k)$, the relays within $\mathcal{R}_{q} \cap \mathcal{R}$ transmit their messages to $D$ simultaneously. The received signal at $D$ is

$$
y_{D}[m+q]=\sum_{R_{l} \in \mathcal{R}_{q} \cap \mathcal{R}} \sqrt{\rho} h_{R_{l}, D} x_{R_{l}}+n_{D}[m+q] .
$$

Clearly, in the case where $m=M$ and $k=K$ all the sources and relays transmit orthogonally. For direct sourcedestination communication without relaying, it is well known that the non-orthogonal transmission among all the sources is more spectrally efficient than the orthogonal transmission and it provides the optimized DMT. We can also adopt such an MA protocol in the considered coded multiple-source multiplerelay network. In this case all the sources (relays) transmit non-orthogonally in the broadcasting (forwarding) phase such that $m=k=1$. In what follows, we term these two straightforward special cases orthogonal MDS-FFNC protocol and non-orthogonal MDS-FFNC protocol respectively.

\section{DMT ANALYSIS}

During each time slot $D$ applies SIC to decode the messages from the related transmitting cluster. If any $M$ out of its $M+$ $|\mathcal{R}|$ received messages are correctly decoded, $D$ can recover the source message set $\mathcal{I}_{S}$. Otherwise, the transmission fails.

According to the Bayes' rule, the overall error probability at $D$ can be expressed as

$$
P_{e}=\sum_{\mathcal{R} \subseteq\left\{R_{1}, \cdots, R_{K}\right\}} P_{\mathcal{R}} \bar{P}_{e \mid \mathcal{R}}
$$

where $P_{\mathcal{R}}$ is the probability that a specific decoding relay set $\mathcal{R}$ occurs, and $\bar{P}_{e \mid \mathcal{R}}$ is the error probability at $D$ (i.e. the probability that the transmission fails) given $\mathcal{R}$. Studying the error behavior, we obtain the DMT performance. We summarize the result in the following theorem.

Theorem 1: Combine the two sets $\mathcal{M}=\left\{M_{1}, \cdots, M_{m}\right\}$ and $\mathcal{K}=\left\{K_{1}, \cdots, K_{k}\right\}$ into a single set $\mathcal{N}=$ $\left\{N_{1}, N_{2}, \cdots, N_{m+k}\right\}$, where $N_{1} \geq N_{2} \geq \cdots \geq N_{m+k}$. If $M_{i}=K_{j}$, the index of $K_{j}$ in $\mathcal{N}$ is smaller than that of $M_{i}$, i.e. place $M_{i}$ behind $K_{j}$. Let $N_{0}=0$. Use $\tau$ to denote the index of $M_{1}$ in $\mathcal{N}$ and use $\zeta$ to denote the index in $\mathcal{N}$ such that $\sum_{i=0}^{\zeta-1} N_{i}<K+1 \leq \sum_{i=0}^{\zeta} N_{i}$. Define $\bar{d}_{\alpha}(r)$ $(\alpha \in\{0,1, \cdots, \zeta-1\})$ and $\bar{d}_{\zeta}(r)$ as (7) and (8) respectively (see next page). The achievable diversity gain of the cluster MDS-FFNC protocol is

$$
d_{\mathcal{M}, \mathcal{K}}(r)=\min _{s \in\{\tau, \tau+1, \cdots, \zeta\}} \bar{d}_{s}(r) .
$$

Proof: See Appendix B.

Theorem 1 implies that the cluster MDS-FFNC protocol achieves the maximal diversity gain $K+1$ (when $r=0$ ) and the maximal multiplexing gain $\frac{1}{(m+k) M_{1}}($ when $d=0$ ). Calculating the entire tradeoff $d_{\mathcal{M}, \mathcal{K}}(r)$ is straightforward. For example, consider a network with 3 sources and 6 relays. If we place the sources into a single cluster and divide the relays into two equal-size clusters, we have $\mathcal{M}=\left\{M_{1}\right\}=\{3\}, \mathcal{K}=$ $\left\{K_{1}, K_{2}\right\}=\{3,3\}$, and $\mathcal{N}=\left\{K_{1}, K_{2}, M_{1}\right\}=\{3,3,3\}$. Since $\tau=\zeta=3$, using (8) and (9) the achievable DMT is

$$
\begin{aligned}
d_{\mathcal{M}, \mathcal{K}}(r)=\bar{d}_{3}(r)= & 3(1-9 r)^{+}+3(1-9 r)^{+} \\
& +\min \left\{(1-3 r)^{+}, 3(1-9 r)^{+}\right\} .
\end{aligned}
$$

We can also divide the relays into three clusters each containing 2 relays. Thus now $\mathcal{N}=\left\{M_{1}, K_{1}, K_{2}, K_{3}\right\}=\{3,2,2,2\}$, $\tau=1$, and $\zeta=3$. Following Theorem 1 , we can see

$$
\begin{gathered}
\bar{d}_{1}(r)=4 \min \left\{(1-4 r)^{+}, 3(1-12 r)^{+}\right\}+3(1-12 r)^{+}, \\
\bar{d}_{2}(r)=2 \min \left\{(1-4 r)^{+}, 3(1-12 r)^{+}\right\} \\
\quad+3(1-12 r)^{+}+2(1-8 r)^{+}, \\
\bar{d}_{3}(r)=3(1-12 r)^{+}+2(1-8 r)^{+}+2(1-8 r)^{+} .
\end{gathered}
$$

As a result, the achievable DMT is expressed as follows ,

$$
\begin{aligned}
d_{\mathcal{M}, \mathcal{K}}(r) & =\min \left\{\bar{d}_{1}(r), \bar{d}_{2}(r), \bar{d}_{3}(r)\right\} \\
& =\left\{\begin{array}{cl}
3(1-12 r)^{+}+4(1-8 r)^{+} & 0 \leq r \leq \frac{1}{14} \\
15(1-12 r)^{+} & \frac{1}{14} \leq r \leq \frac{1}{12}
\end{array} .\right.
\end{aligned}
$$

These two DMT curves are displayed in Fig. 2. To show the spectral efficiency of employing MDS-FFNC in multisource multi-relay networks, we plot the DMT performance of applying the standard orthogonal DF relaying protocol [2] in the network. To obtain the maximal diversity gain $K+1$, the standard protocol normally requires $K+1$ time slots to finish each source message's transmission: one time slot for the source to broadcast the message and $K$ time slots for the relays to successively forward this message to $D$ (using the same channel code as the source). The destination applies maximum ratio combining (MRC) to its received signals to recover the source message. The transmission of $\mathcal{I}_{S}$ is completed in a total of $M(K+1)$ time slots and the following DMT is attained,

$$
d_{s}(r)=(K+1)(1-M(K+1) r)^{+} .
$$

The maximal achievable multiplexing gain is only $\frac{1}{M(K+1)}$.

In addition, we also plot the DMT performance of the orthogonal and non-orthogonal MDS-FFNC protocols in Fig. 2. Clearly, when the multiplexing gain $r=0$, the diversity gain $K+1$ can be attained by any clustering strategy (i.e. any choice of $\mathcal{M}$ and $\mathcal{K}$ ). In this sense, different clustering strategies are equally good and cannot be distinguished. However, when $r>0$, choosing different $\mathcal{M}$ and $\mathcal{K}$ may lead to quite different diversity performances. In the following section, we 


$$
\begin{array}{r}
\bar{d}_{\alpha}(r)=\left(K+1-\sum_{i=0}^{\alpha} N_{i}\right) \min \left\{(1-(m+k) r)^{+}, M_{1}\left(1-(m+k) M_{1} r\right)^{+}\right\}+\sum_{i=0}^{\alpha} N_{i}\left(1-(m+k) N_{i} r\right)^{+} \\
\bar{d}_{\zeta}(r)=\min \left\{\left(K+1-\sum_{i=0}^{\zeta-1} N_{i}\right)\left(1-(m+k)\left(K+1-\sum_{i=0}^{\zeta-1} N_{i}\right) r\right)^{+}, N_{\zeta}\left(1-(m+k) N_{\zeta} r\right)^{+}\right\}+\sum_{i=0}^{\zeta-1} N_{i}\left(1-(m+k) N_{i} r\right)^{+}
\end{array}
$$

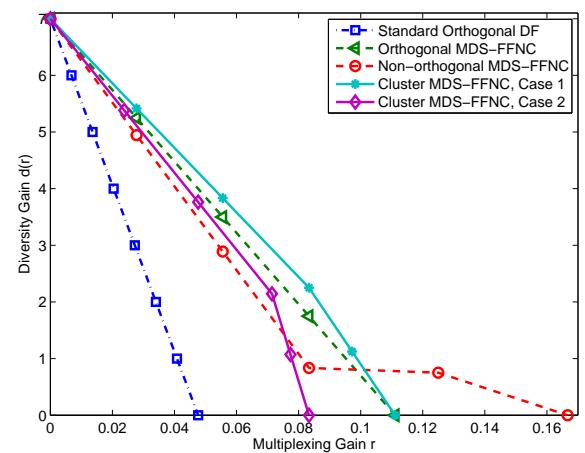

Fig. 2. DMT comparison when $M=3$ and $K=6$. The cluster MDS-FFNC protocol cases 1 and 2 denote the cases where the relays are divided into 2 and 3 equal-size clusters, respectively.

will discuss how to cluster the sources and relays in order to optimize the achievable DMT performance.

\section{Clustering Strategies}

For any clustering strategy (i.e. given $\mathcal{M}=\left\{M_{1}, \cdots, M_{m}\right\}$ and $\mathcal{K}=\left\{K_{1}, \cdots, K_{k}\right\}$ ), the achievable DMT performance of the cluster MDS-FFNC protocol $d_{\mathcal{M}, \mathcal{K}}(r)$ can be calculated following Theorem 1. Consequently, for a given value of $r$, it is desired that the sources and the relays should be clustered such that the achievable diversity gain is optimized. In other words, the values of $m, k, M_{1}, \cdots, M_{m}, K_{1}, \cdots, K_{k}$ should be designed to achieve the following diversity gain

$$
d_{M, K}^{*}(r)=\max _{m, k, M_{1}, \cdots, M_{m}, K_{1}, \cdots, K_{k}} d_{\mathcal{M}, \mathcal{K}}(r) .
$$

It is difficult to find the closed form of (10). However, for fixed $m$ and $\mathcal{K}$, it is possible to design the clustering strategy for the sources to attain the highest value of $d_{\mathcal{M}, \mathcal{K}}(r)$. We summarize the result as the following theorem.

Theorem 2: For an $M$-source $K$-relay network, given $m$ and $\mathcal{K}, d_{\mathcal{M}, \mathcal{K}}(r)$ is optimized when the sources are evenly divided, i.e. $M_{1}=M_{2}=\cdots=M_{p}=\left\lceil\frac{M}{m}\right\rceil$ and $M_{p+1}=$ $M_{p+2}=\cdots=M_{m}=\left\lfloor\frac{M}{m}\right\rfloor$, where $p=m+M-m\left\lceil\frac{M}{m}\right\rceil$.

Proof: If the sources are not evenly divided, one can always find two clusters, say the $x$ th and the $y$ th clusters $(1 \leq$ $x<y \leq m$ ), so that moving one source from the $x$ th cluster to the $y$ th cluster does not change the indices $x$ and $y$. Consequently, the set $\mathcal{N}=\left\{N_{1}, \cdots, N_{p}, \cdots, N_{q}, \cdots, N_{m+k}\right\}$ is changed to $\mathcal{N}^{\prime}=\left\{N_{1}, \cdots, N_{p}-1, \cdots, N_{q}+1, \cdots, N_{m+k}\right\}$, where there are five situations regarding $p$ and $q: 1) \tau \leq$ $p<q<\zeta$, 2) $\tau \leq p<q=\zeta$, 3) $\tau \leq p<\zeta<q$,

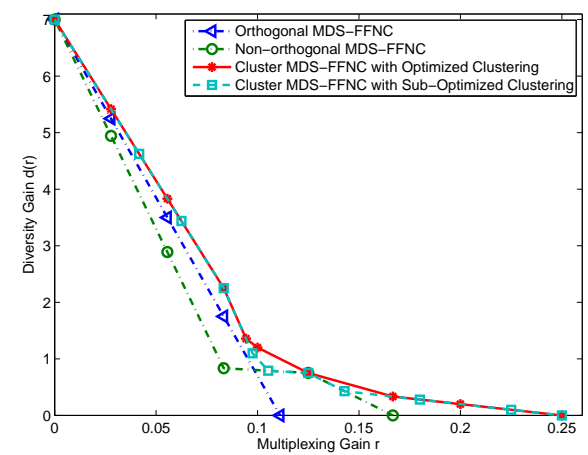

Fig. 3. DMT Comparison when $M=3$ and $K=6$.

4) $\tau \leq p=\zeta<q$, and 5) $\tau \leq \zeta<p<q$. Use $\bar{d}_{s}(r)$ $(\tau \leq s \leq \zeta)$ to denote the diversity gains (expressed as (7) and (8)) for the case corresponding to $\mathcal{N}$ (i.e. before one source is moved). And use $\bar{d}_{s}^{\prime}(r)$ to denote the diversity gains for the case corresponding to $\mathcal{N}^{\prime}$. For each of the five situations, following the proof of Theorem 1 in Appendix B we can prove $\min _{s \in\{\tau, \cdots, \zeta\}} \bar{d}_{s}(r) \leq \min _{s \in\{\tau, \cdots, \zeta\}} \bar{d}_{s}^{\prime}(r)$. Therefore, moving one source from the larger cluster $x$ to the smaller cluster $y$ increases the diversity gain. When the sources are evenly divided, the diversity gain achieves the maximal value. Due to limited space, here we omit the detailed proof, which can be found in [12].

For fixed $m$ and $k$, evenly dividing the relays does not always provide the optimized performance. In general, for a given value of $r$, a brute-force searching for all possible $m$ and $\mathcal{K}$ is required to find the best clustering strategy such that the optimized diversity gain (10) can be attained.

To reduce calculation complexity, one can choose evenly dividing the relays to obtain a sub-optimized performance. In other words, set $K_{1}=K_{2}=\cdots=K_{q}=\left\lceil\frac{K}{k}\right\rceil$ and $K_{q+1}=$ $K_{q+2}=\cdots=K_{k}=\left\lfloor\frac{K}{k}\right\rfloor$, where $q=k+K-k\left\lceil\frac{K}{k}\right\rceil$. A total of $M \times K$ different values of $d_{\mathcal{M}, \mathcal{K}}(r)$ need to be calculated and compared for a given $r$. We compare the DMT performance for two example networks in Fig. 3 and Fig. 4. The advantages of choosing appropriate clustering strategies are clearly seen.

\section{CONCLUSION}

We have proposed a novel transmission protocol for a coded multi-source multi-relay network where a class of MDSFFNCs are employed in the relays. The sources and relays are divided into clusters. The nodes within the same cluster 


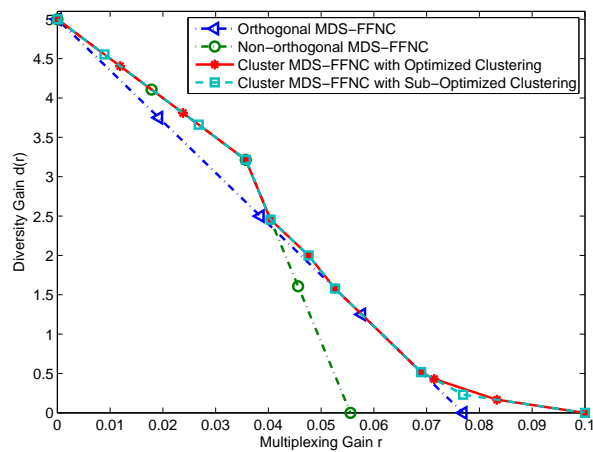

Fig. 4. DMT Comparison when $M=9$ and $K=4$.

transmit non-orthogonally while the nodes from different clusters transmit orthogonally. It has been shown that for a given value of multiplexing gain, the achievable diversity gain can be optimized through the most appropriate clustering strategy.

\section{APPENDIX A \\ PRELIMINARY RESULTS}

We first provide some preliminary results for the proof of Theorem 1. In what follows, we use $\mathcal{P}\{A\}$ to denote the probability that event $A$ occurs. $\doteq$ denotes the exponential equality operator [11]: $X \doteq \rho^{b}$ is equivalent to $b=\lim _{\rho \rightarrow \infty} \frac{\log X}{\log \rho}$. Operators $\dot{<}$ and $\dot{>}$ are defined similarly.

Consider a single-antenna multi-user communication system where $N$ transmitting nodes intend to send independent messages to a common receiving node. Assume that all the transmitting nodes communicate with the receiving node nonorthogonally and the receiving node applies SIC to decode the transmit messages. Use $\mathcal{G} \subseteq\{1,2, \cdots, N\}$ to denote the subset of transmitting nodes whose messages can be successfully decoded and use $\mathcal{B}=\mathcal{G}^{c} \subseteq\{1,2, \cdots, N\}$ to denote the remaining subset of transmitting nodes whose messages cannot be decoded without errors. We have [13]

$$
\begin{gathered}
\log \left(1+\frac{\sum_{i \in \mathcal{S}} \rho\left|h_{i}\right|^{2}}{1+\sum_{i \in \mathcal{B}} \rho\left|h_{i}\right|^{2}}\right)>|\mathcal{S}| R, \quad \forall \mathcal{S} \subseteq \mathcal{G}, \\
\log \left(1+\frac{\sum_{i \in \mathcal{T}} \rho\left|h_{i}\right|^{2}}{1+\sum_{i \in \mathcal{T}_{c}} \rho\left|h_{i}\right|^{2}}\right)<|\mathcal{T}| R, \quad \forall \mathcal{T} \subseteq \mathcal{B},
\end{gathered}
$$

where $\mathcal{T}_{c}=\mathcal{T}^{c} \cap \mathcal{B}$. Assuming $R=\bar{R}$, we can calculate the probability that the receiving node can decode $N-n$ transmitting nodes' messages and it cannot decode $n$ transmitting nodes' messages as

$$
\begin{aligned}
P_{n} & \leq \mathcal{P}\left\{\mathcal{C}\left(\frac{\sum_{i \in \mathcal{T}}\left|h_{i}\right|^{2}}{1+\sum_{i \in \mathcal{T}_{c}} \rho\left|h_{i}\right|^{2}}\right)<|\mathcal{T}| \bar{R}, \mathcal{T}=\mathcal{B}\right\} \\
& =\mathcal{P}\left\{\log \left(1+\sum_{i=N-n+1}^{N} \rho\left|h_{i}\right|^{2}\right)<n \bar{R}\right\} \doteq \rho^{-n(1-n r)^{+}}
\end{aligned}
$$

where $\mathcal{C}(x)=\log (1+\rho x)$, and without loss of generality we assume $\mathcal{G}=\{1,2, \cdots, N-n\}$ and $\mathcal{B}=\{N-n+1, N-$ $n+2, \cdots, N\}$.
Since $\min \left\{n(1-n r)^{+},(n+1)(1-(n+1) r)^{+}, \cdots, N(1-\right.$ $\left.N r)^{+}\right\}=\min \left\{n(1-n r)^{+}, N(1-N r)^{+}\right\}$[14], we can calculate the probability that the receiving node decodes at most $N-n$ transmitting nodes' messages (i.e. it cannot decode at least $n$ transmitting nodes' messages) as

$$
P_{n}^{+}=\sum_{s=n}^{N} P_{s} \dot{\leq} \sum_{s=n}^{N} \rho^{-s(1-s r)^{+}} \doteq \rho^{-\min \left\{n(1-n r)^{+}, N(1-N r)^{+}\right\}} .
$$

Now we consider another network where one receiving node listens to two clusters of transmitting nodes with $W_{1}$ and $W_{2}\left(W_{1} \geq W_{2}\right)$ nodes respectively. The nodes within each cluster transmit simultaneously but the transmissions of the two clusters are orthogonal to each other. We want to find $\tilde{P}_{V}^{+}$, the probability that the receiving node cannot decode at least $V$ transmitting nodes' messages. Clearly, $\tilde{P}_{V}^{+}=\sum_{s=\left(V-W_{2}\right)}^{W_{1}} p_{1, s} p_{2, V-s}^{+}$, where $p_{1, s}$ is the probability that the receiving node cannot decode the messages from $s$ nodes within cluster 1 , and $p_{2, V-s}^{+}$is the probability that the receiving node cannot decode the transmit messages from at least $V-s$ nodes within cluster 2 . Let $f(a, b)=a(1-b a r)^{+}$. We can express $\tilde{P}_{V}^{+}$as $\tilde{P}_{V}^{+} \doteq \rho^{-\tilde{d}_{V}}$, where

$\tilde{d}_{V}=\min _{\left(V-W_{2}\right)^{+} \leq s \leq W_{1}}\left\{f(s, 2)+\min \left\{f(V-s, 2), f\left(W_{2}, 2\right)\right\}\right\}$.

It is not difficult to show that when $V \leq W_{1}$

$$
\tilde{d}_{V}=\min \left\{f(V, 2), f\left(W_{1}, 2\right)\right\}
$$

and when $W_{1}<V$

$$
\tilde{d}_{V}=f\left(W_{1}, 2\right)+\min \left\{f\left(V-W_{1}, 2\right), f\left(W_{2}, 2\right)\right\} .
$$

If the receiving node listens to three orthogonal transmitting clusters with $W_{1}, W_{2}$, and $W_{3}$ concurrent transmitting nodes respectively $\left(W_{1} \geq W_{2} \geq W_{3}\right)$, the probability that the receiving node cannot decode the messages of at least $V$ nodes can be shown as $\tilde{P}_{V}^{+} \doteq \rho^{-\tilde{d}_{V}}$, where when $V \leq W_{1}$

$$
\tilde{d}_{V}=\min \left\{f(V, 3), f\left(W_{1}, 3\right)\right\} .
$$

When $W_{1}<V \leq W_{1}+W_{2}$

$$
\tilde{d}_{V}=f\left(W_{1}, 3\right)+\min \left\{f\left(V-W_{1}, 3\right), f\left(W_{2}, 3\right)\right\} .
$$

Finally, when $W_{1}+W_{2}<V$

$\tilde{d}_{V}=f\left(W_{1}, 3\right)+f\left(W_{2}, 3\right)+\min \left\{f\left(V-W_{1}-W_{2}, 3\right), f\left(W_{3}, 3\right)\right\}$.

Similarly, we can consider a more general case with $w$ orthogonal transmitting clusters, containing $W_{1}, W_{2}, \cdots, W_{w}$ concurrent transmitting nodes respectively $\left(W_{1} \geq W_{2} \geq \cdots \geq\right.$ $\left.W_{w}\right)$. We pick $V$ nodes, started from the largest cluster first, then from the second largest cluster, and so on, until the $i$ th largest cluster, i.e. $\sum_{j=1}^{i-1} W_{j}<V \leq \sum_{j=1}^{i} W_{j}$. The probability that the receiving node cannot decode the messages transmitted by at least $V$ nodes is dominated by the probability that the receiving node cannot decode the messages transmitted by all the nodes from the first $i-1$ largest clusters and at least $\left(V-\sum_{j=1}^{i-1} W_{j}\right)$ nodes from the $i$ th cluster. 


\section{APPENDIX B \\ PROOF OF THEOREM 1}

The overall error probability (6) can be re-written as

$$
P_{e}=\sum_{u_{1}=0}^{K_{1}} \cdots \sum_{u_{k}=0}^{K_{k}} P_{u_{1}, \cdots, u_{k}} \bar{P}_{e \mid u_{1}, \cdots, u_{k}},
$$

in which $P_{u_{1}, \cdots, u_{k}}$ is the probability that $u_{1}, \cdots, u_{k}$ relays within clusters $\mathcal{R}_{1}, \cdots, \mathcal{R}_{k}$ are activated to assist the sources respectively, and $\bar{P}_{e \mid u_{1}, \cdots, u_{k}}$ is the associated error probability. Assuming $U=\sum_{q=1}^{k} u_{q}$, since $M_{1} \geq M_{2} \geq \cdots \geq M_{m}$, we have $P_{u_{1}, \cdots, u_{k}} \doteq \rho^{-\hat{d}(U)}$, where

$$
\hat{d}(U)=(K-U) \min \left\{f(1, m+k), f\left(M_{1}, m+k\right)\right\} .
$$

The transmission fails if the messages transmitted by at least $U+1$ nodes cannot be decoded by $D$. We reorder the set $\left\{M_{1}, \cdots, M_{m}, u_{1}, \cdots, u_{k}\right\}$ into set $\hat{\mathcal{N}}=\left\{n_{1}, n_{2}, \cdots, n_{m+k}\right\}$ where $n_{1} \geq n_{2} \geq \cdots \geq n_{m+k}$. Let $\hat{g}(a)=\sum_{i=1}^{a} n_{i}$ and use $\mu$ to denote the index such that $\hat{g}(\mu-1)<U+1 \leq \hat{g}(\mu)$. The results we provided in Appendix A implies that $\bar{P}_{e \mid u_{1}, \cdots, u_{k}}$ is dominated by the probability that $D$ cannot decode the messages transmitted by all the first $\mu-1$ clusters and at least $(U+1-\hat{g}(\mu-1))$ nodes from cluster $\mu$, i.e. $\bar{P}_{e \mid u_{1}, \cdots, u_{k}} \doteq \rho^{-\bar{d}\left(u_{1}, \cdots, u_{k}\right)}$, where $\bar{d}\left(u_{1}, \cdots, u_{k}\right)=$ $\min \left\{f(U+1-\hat{g}(\mu-1), m+k), f\left(n_{\mu}, m+k\right)\right\}+$ $\sum_{i=1}^{\mu-1} f\left(n_{i}, m+k\right)$.

Therefore, given $\mathcal{M}$ and $\mathcal{K}$, the cluster MDS-FFNC protocol achieves the DMT

$d_{\mathcal{M}, \mathcal{K}}(r)=\min _{u_{q} \in\left\{0, \cdots, K_{q}\right\}, \forall q \in\{1, \cdots, k\}}\left\{\hat{d}(U)+\bar{d}\left(u_{1}, \cdots, u_{k}\right)\right\}$.

Moreover, we combine the sets $\mathcal{M}=\left\{M_{1}, \cdots, M_{m}\right\}$ and $\mathcal{K}=\left\{K_{1}, \cdots, K_{k}\right\}$ into one set $\mathcal{N}=\left\{N_{1}, N_{2}, \cdots, N_{m+k}\right\}$ where $N_{1} \geq N_{2} \geq \cdots \geq N_{m+k}$. If any $M_{i}=K_{j}$, we place $M_{i}$ behind $K_{j}$. The index of $M_{1}$ in $\mathcal{N}$ is $\tau$. Let $g(a)=$ $\sum_{i=1}^{a} N_{i}$. Denote $\xi$ and $\zeta$ the indices in $\mathcal{N}$ such that $g(\xi-1)<$ $U+1 \leq g(\xi)$ and $g(\zeta-1)<K+1 \leq g(\zeta)$. Clearly, $1 \leq \xi \leq \zeta$ and $\zeta \geq \tau$.

If $\xi<\tau$ and $U+1-g(\xi-1) \leq M_{1}$, we define

$$
\begin{gathered}
\bar{d}(U)=\min \left\{f(U+1-g(\xi-1), m+k), f\left(M_{1}, m+k\right)\right\} \\
+\sum_{i=1}^{\xi-1} f\left(N_{i}, m+k\right) .
\end{gathered}
$$

Otherwise, if $\xi<\tau$ and $U+1-g(\xi-1)>M_{1}$, define

$$
\begin{gathered}
\bar{d}(U)=\sum_{i=1}^{\xi-1} f\left(N_{i}, m+k\right)+f(U-g(\xi-1), m+k) \\
+\min \left\{f(1, m+k), f\left(M_{1}, m+k\right)\right\} .
\end{gathered}
$$

On the other hand, if $\xi \geq \tau$, we define

$$
\begin{gathered}
\bar{d}(U)=\min \left\{f(U+1-g(\xi-1), m+k), f\left(N_{\xi}, m+k\right)\right\} \\
+\sum_{i=1}^{\xi-1} f\left(N_{i}, m+k\right) .
\end{gathered}
$$

Let $d(U)=\hat{d}(U)+\bar{d}(U)$. It is not difficult to prove that (17) can be re-written as

$$
d_{\mathcal{M}, \mathcal{K}}(r)=\min _{U \in\{0, \cdots, K\}} d(U) .
$$

Using (18)-(20) it can be seen that $d(U)$ monotonously decreases with $U$ when $0 \leq U \leq g(\tau)-1$. If $\zeta=\tau$, we directly substitute $U=K$ into (20) and obtain the diversity gain.

If $\zeta>\tau$, using (20) we can easily see that

$$
\begin{gathered}
\min _{U \in\{g(\alpha)-1, g(\alpha), \cdots, g(\alpha+1)-1\}} d(U) \\
=\min \{d(g(\alpha)-1), d(g(\alpha+1)-1)\}, \tau \leq \alpha \leq \zeta-2, \\
\min _{U \in\{g(\zeta-1)-1, g(\zeta-1), \cdots, K\}} d(U)=\min \{d(g(\zeta-1)-1), d(K)\} .
\end{gathered}
$$

As a result, the achievable diversity gain can be expressed by

$$
d_{\mathcal{M}, \mathcal{K}}(r)=\min _{U \in\{g(\tau)-1, g(\tau+1)-1, \cdots, g(\zeta-1)-1, K\}} d(U) .
$$

The proof is complete.

\section{ACKNOWLEDGMENT}

The research was supported by the Swedish Foundation for Strategic Research, Swedish Research Council (VR), and VINNOVA.

\section{REFERENCES}

[1] J. N. Laneman, D. N. C. Tse, and G. W. Wornell, "Cooperative diversity in wireless networks: Efficient protocols and outage behavior," IEEE Trans. Inform. Theory, vol. 50, no. 12, pp. 3062-3080, Dec. 2004.

[2] J. N. Laneman and G. W. Wornell, "Distributed space-time-coded protocols for exploiting cooperative diversity in wireless networks," IEEE Trans. Inform. Theory, vol. 49, no. 10, pp. 2415-2425, Oct. 2003

[3] S. Li, R. W. Yeung, and N. Cai, "Linear network coding," IEEE Trans. Inform. Theory, vol. 49, pp. 371-381, Feb. 2003.

[4] R. Koetter and M. Medard, "An algebraic approach to network coding," IEEE/ACM Trans. on Networking, vol. 11, pp. 782-795, Oct. 2003.

[5] C. Hausl, F. Schreckenbach, I. Oikonomidis, and G. Bauch, "Iterative network and channel decoding on a tanner graph," in 43rd Annual Allerton Conference on Communication, Control, and Computing, Monticello, USA, Sep. 2005.

[6] S. Yang and R. Koetter, "Network coding over a noisy relay: A belief propagation approach," in IEEE International Symposium on Information Theory (ISIT), Toronto, Canada, Jul. 2008.

[7] M. Xiao and M. Skoglund, "Design of network codes for multiple-user multiple-relay wireless networks," in IEEE International Symposium on Information Theory (ISIT), Seoul, Korea, Jun. 2009.

[8] M. Xiao, J. Kliewer, and M. Skoglund, "Design of network codes for multiple-user multi-relay wireless networks," revised version submitted to IEEE Trans. Wireless Commun., Aug. 2010.

[9] R. W. Yeung, S.-Y. Li, N. Cai, and Z. Zhang, Network Coding Theory. MA, USA: NOW publishers, Inc, Hanover, 2006.

[10] F. J. Macwilliams and N. J. A. Sloane, The Theory of Error-Correcting Codes. New York: North-Holland, 1978.

[11] L. Zheng and D. N. C. Tse, "Diversity and multiplexing: A fundamental tradeoff in multiple-antenna channels," IEEE Trans. Inform. Theory, vol. 49, no. 5, pp. 1073-1096, May 2003.

[12] C. Wang, M. Xiao, and M. Skoglund, "Diversity-multiplexing tradeoff analysis of coded multi-user relay networks," submitted to IEEE Trans. Commun., Sep. 2010.

[13] R. Narasimhan, "Individual outage rate regions for fading multiple access channels," in IEEE International Symposium on Information Theory (ISIT), Nice, France, Jun. 2007.

[14] D. N. C. Tse, P. Viswanath, and L. Zheng, "Diversity-multiplexing tradeoff in multiple-access channels," IEEE Trans. Inform. Theory, vol. 50, no. 9, pp. 1859-1874, Sep. 2004. 